PROCEEDINGS OF THE

AMERICAN MATHEMATICAL SOCIETY

Volume 135, Number 9, September 2007, Pages 2893-2898

S 0002-9939(07)08823-5

Article electronically published on May 8, 2007

\title{
MAPPING PROPERTIES OF ANALYTIC FUNCTIONS ON THE DISK
}

\author{
PIETRO POGGI-CORRADINI
}

(Communicated by Juha M. Heinonen)

\begin{abstract}
There is a universal constant $0<r_{0}<1$ with the following property. Suppose that $f$ is an analytic function on the unit disk $\mathbb{D}$, and suppose that there exists a constant $M>0$ so that the Euclidean area, counting multiplicity, of the portion of $f(\mathbb{D})$ which lies over the disk $D(f(0), M)$, centered at $f(0)$ and of radius $M$, is strictly less than the area of $D(f(0), M)$. Then $f$ must send $r_{0} \overline{\mathbb{D}}$ into $D(f(0), M)$. This answers a conjecture of Don Marshall.
\end{abstract}

\section{INTRODUCTION}

Let $f$ be analytic on $\mathbb{D}, f(0)=0$. For $M>0$, let

$$
\Omega(M)=\{z \in \mathbb{D}:|f(z)|<M\}=\mathbb{D} \cap f^{-1}(D(0, M)) .
$$

Write $A(M)$ for the Euclidean area of $f(\Omega(M))$ counting multiplicity, i.e.,

$$
A(M)=\int_{\Omega(M)}\left|f^{\prime}(z)\right|^{2} d A(z) .
$$

Theorem 1.1. There is a universal constant $r_{0}>0$ such that whenever $f$ is analytic on $\mathbb{D}$ with $f(0)=0$, and whenever $M>0$ is such that $A(M)<\pi M^{2}$, then $f\left(r_{0} \mathbb{D}\right) \subset D(0, M)$.

Theorem 1.1 was conjectured in Mar89] p. 135. As Marshall notes, if $f$ is oneto-one, then two applications of the $1 / 4$-Koebe Theorem suffice. In fact, suppose $f$ is conformal on $\mathbb{D}$ with $f(0)=0$ and $M$ is such that $A(M)<\pi M^{2}$. Then, $D(0, M) \backslash f(\mathbb{D})$ must be non-empty. So if $R>0$ is the largest radius for which $D(0, R) \subset f(\mathbb{D})$, then $R<M$. By Koebe, $R \geq\left|f^{\prime}(0)\right| / 4$. Now consider $f^{-1}$ restricted to $D(0, R)$. By Koebe again, $f^{-1}(D(0, R))$ must contain a disk centered at the origin of radius $R\left|\left(f^{-1}\right)^{\prime}(0)\right| / 4 \geq 1 / 16$, i.e., $f\left(\frac{1}{16} \mathbb{D}\right) \subset D(0, R) \subset D(0, M)$. Therefore Theorem 1.1 can be thought of as a generalization to many-to-one maps of "two applications of Koebe".

Before proving Theorem 1.1, we will reformulate it in a couple of different ways. For $0<r<1$, consider the growth function

$$
\mathcal{A}(r)=\frac{A(M(r))}{\pi M(r)^{2}},
$$

Received by the editors January 3, 2006 and, in revised form, June 1, 2006.

2000 Mathematics Subject Classification. Primary 30C55.

(C)2007 American Mathematical Society 
where $M(r)=\max _{|z|=r}|f(z)|$. So $\mathcal{A}(r)$ is a sort of Euclidean Mean Covering. It is larger than the usual (Euclidean) Ahlfors growth function. Then Theorem 1.1 is equivalent to the following statements:

Corollary 1.2. There is a universal radius $r_{0}>0$ so that for every $f$ analytic in $\mathbb{D}$ with $f(0)=0$, and for every $0<r<r_{0}$,

$$
\mathcal{A}(r) \geq 1 \text {. }
$$

Corollary 1.3. There is a universal radius $r_{0}>0$ so that for every $f$ analytic in $\mathbb{D}$ with $f(0)=0$, and for every $0 \leq M \leq \max _{|z|=r_{0}}|f(z)|$,

$$
A(M) \geq \pi M^{2} .
$$

Proof of Corollary 1.2; Let $r_{0}$ be the constant given in Theorem 1.1. Suppose that for some $r<r_{0}, \mathcal{A}(r)<1$, i.e., $A(M(r))<\pi M(r)^{2}$. Then, by Theorem 1.1. $f\left(r_{0} \mathbb{D}\right) \subset D(0, M(r))$. Hence $M\left(r_{0}\right) \leq M(r)$, but this contradicts the maximum principle.

Corollary 1.3 is proved similarly.

We end this introduction with a remark and a question related to Theorem 1.1.

Remark 1.4. The proof of Theorem 1.1 provided below breaks down for meromorphic functions when the "Ahlfors island argument" is used. Indeed, here is a simple counterexample when $f$ is allowed to have poles. The function

$$
f(z)=\frac{z}{\epsilon+2 z}
$$

maps $\mathbb{D}$ conformally onto the complement in the extended complex plane of a small disk containing the point $1 / 2, f(0)=0$, and choosing $M=1$ in Theorem 1.1 we have $A(1)<\pi$, but $f(-\epsilon / 3)=-1$, for all $\epsilon>0$.

Remark 1.5. To see that the bound on $A(M)$ in Theorem 1.1 is needed and cannot be replaced with the condition that $D(0, M) \backslash f(\mathbb{D}) \neq \emptyset$, consider the following example:

$$
f_{n}(z)=1-\exp \left(\frac{2 z n}{1-z}\right) .
$$

Then $f_{n}(0)=0$, and $f_{n}$ omits a small disk centered at 1 . Yet for a fixed $0<r<1$, $f_{n}(r) \rightarrow-\infty$ as $n \rightarrow \infty$.

Question 1.6. Does a form of Theorem 1.1 still hold for quasi-regular maps of the unit ball of $\mathbb{R}^{n}, n \geq 2$ ?

Since the main tool in the proof of Theorem 1.1 is modulus of path families, it is safe to expect this phenomenon to persist in the quasi world. However, a few initial attempts on our part failed when trying to lift paths.

\section{A proof of Theorem 1.1}

Without loss of generality $f$ is analytic in $\overline{\mathbb{D}}$ (otherwise consider $f(t z)$ with $t$ close to 1$)$. Notice that $D(0, M) \backslash f(\mathbb{D}) \neq \emptyset$, because otherwise we would have $A(M) \geq \pi M^{2}$. So we can pick a point $\zeta \in D(0, M) \backslash f(\mathbb{D})$, and we can rotate and dilate the image so as to have $\zeta>0$ and $M=1$. We let $G=G(1)$ be the component of $\Omega(1)$ which contains the origin and we let $r$ be the largest radius so that $r \mathbb{D} \subset G$. Then $r \overline{\mathbb{D}} \cap \partial G \neq \emptyset$. Hence $0 \in f(r \overline{\mathbb{D}}) \subset \overline{D(0,1)}$ and $f(r \overline{\mathbb{D}}) \cap \partial D(0,1) \neq \emptyset$. Consider 
a ray $[0, a]$ connecting 0 to a point $a$ in $r \partial \mathbb{D} \cap \partial G$. Then $E=f([0, a])$ is a curve in $f(r \mathbb{D})$ connecting 0 to $\partial D(0,1)$.

Several cases arise. Assume first that $0<\zeta<1 / 4$; then we consider the family of circles centered at $\zeta$ with radius $u$ and $1 / 4<u<3 / 4$. Each one of these circles separates 0 from $\partial D(0,1)$, so each one must intersect $E$. Since $E=E(s)=f(s a)$, $0<s<1$ is a piecewise analytic arc, we can find finitely many open intervals $J_{n} \subset[0,1], n=1, . ., N$, so that $h_{n}(s)=|E(s)-\zeta|$ is an increasing function on each interval $J_{n}$, the ranges $I_{n}=h_{n}\left(J_{n}\right)$ are disjoint and their union $\bigcup_{n} I_{n}$ spans the whole interval $[1 / 4,3 / 4]$ except for possibly finitely many points. Let $F_{n}=\{s a$ : $\left.s \in J_{n}\right\}$ and $E_{n}=\left\{f(s a): s \in J_{n}\right\}$. Then every point $w_{u} \in E_{n} \cap\{|w-\zeta|=u\}$ is hit schlichtly by the corresponding point $z_{u} \in F_{n}$, and a branch of $f^{-1}$ is well defined in a neighborhood of $E_{n}$ such that $f^{-1}\left(w_{u}\right)=z_{u}$. Also, such a branch can be analytically continued along $\gamma_{u}(t)=\zeta+u e^{i t}$, for $t>\theta_{u}$, where $\theta_{u}=\operatorname{Arg}\left(w_{u}\right)$ plus a multiple of $2 \pi$ to be determined later. In fact, $\theta_{u}$ can be chosen to be a continuous function in $u$ on each interval $I_{n}$. We extend the definition of $\gamma_{u}(t)$ for $t \in\left[\theta_{u}, T_{u}\right)$, where $T_{u}$ is the largest possible value so as to be able to analytically continue $f^{-1}$ along $\gamma_{u} \mid\left[\theta_{u}, T\right]$ for every $T<T_{u}$, with values in $\mathbb{D}$. Let $\alpha_{u}(t)$ be the lifted paths $f^{-1}\left(\gamma_{u}(t)\right)$. We claim that $\alpha_{u}$ is a simple curve. If not, there are $t_{0} \neq t_{1}$ such that

$$
f^{-1}\left(\gamma_{u}\left(t_{0}\right)\right)=f^{-1}\left(\gamma_{u}\left(t_{1}\right)\right) .
$$

Moreover, $t_{0}$ and $t_{1}$ can be chosen so that the curve $f^{-1}\left(\gamma_{u}(t)\right)$ between $t_{0}$ and $t_{1}$ is a simple closed curve, and hence bounds a region, which is relatively compact in $\mathbb{D}$ (an "island" in Ahlfors terminology). The image of such a region has boundary in the circle $\{w:|w-\zeta|=u\}$ and hence must wind at least once around some and therefore all points inside the circle. So $\zeta$ must be in the image of $f$, by the argument principle, but this is not the case. So $\alpha_{u}$ is a simple curve. As a corollary we find that $\left|\alpha_{u}(t)\right|$ must tend to 1 as $t$ tends to $T_{u}$.

We will estimate the modulus $\operatorname{Mod}(\Gamma)$ of the family $\Gamma$ of all such lifted paths $\alpha_{u}$. Recall that $\operatorname{Mod}(\Gamma)$ is the infimum of $\int_{\mathbb{C}} \rho^{2}(z) d A(z)$ over all admissible $\rho$ 's, i.e., positive Borel measurable functions $\rho$ on $\mathbb{C}$ with the property that

$$
\int_{\alpha} \rho d s \geq 1
$$

for every path $\alpha \in \Gamma$.

Since $\Gamma$ is a subfamily of all paths connecting $r \overline{\mathbb{D}}$ to $\partial \mathbb{D}$, by the monotonicity of modulus, we get

$$
\operatorname{Mod}(\Gamma) \leq \frac{2 \pi}{\log (1 / r)} .
$$

On the other hand, $g(z)=\log (f(z)-\zeta)$ can be defined to be an analytic function on $\mathbb{D}$. So by Oht70, $\S 2.4$ or Ric93, Theorem 8.1 (Poletskii's Inequality),

$$
\operatorname{Mod}(\Gamma) \geq \operatorname{Mod}(g \Gamma)
$$

where $g \Gamma$ is the family of paths of the form $g\left(\alpha_{u}\right)$. In particular, each path in $g \Gamma$ is a vertical segment of the form

$$
\beta_{u}(t)=\log u+i t ; 1 / 4<u<3 / 4, u \notin Z, \theta_{u}+2 k \pi<t<T_{u}+2 k \pi
$$

for some $k=k(u) \in \mathbb{Z}$. Notice that $k(u)$ can be chosen to be constant for $u$ in an interval $I_{n}$, and then the set $U_{n}=\bigcup_{u \in I_{n}} \beta_{u}$ is a simply connected open set on 
which a branch of $g^{-1}$ is well defined. In particular, the set $V_{n}=\bigcup_{u \in I_{n}} \alpha_{u}$ is also open and simply connected in $\mathbb{D}$ and equals $g^{-1}\left(U_{n}\right)$.

We will obtain a lower bound for $\operatorname{Mod}(g \Gamma)$. Assume that $\rho$ is a positive Borel function on $\mathbb{C}$ which is admissible for $g \Gamma$. Then

$$
1 \leq \int_{\beta_{u}} \rho d t \leq\left(\operatorname{Length}\left(\beta_{u}\right)\right)^{1 / 2}\left(\int_{\beta_{u}} \rho^{2} d t\right)^{1 / 2} .
$$

Now, since $U_{n}$ is an open set, the function $u \mapsto \operatorname{Length}\left(\beta_{u}\right)$ is Borel measurable on each interval $I_{n}$. So changing variables via the co-area formula (see MSZ03 for the co-area formula for Sobolev maps):

$$
\begin{aligned}
\int_{1 / 4}^{3 / 4} \operatorname{Length}\left(\beta_{u}\right) u d u & =\int_{1 / 4}^{3 / 4} \int_{\alpha_{u}}|f(z)-\zeta|\left|g^{\prime}(z)\right| d s d u \\
& =\int_{\cup_{n} V_{n}}\left|f^{\prime}(z)\right|^{2} d A(z) \leq A(1)<\pi .
\end{aligned}
$$

So

$$
\begin{aligned}
\int_{\mathbb{C}} \rho^{2} d u d t & \geq \int_{1 / 4}^{3 / 4} \int_{\beta_{u}} \rho^{2} d t d u \\
& \geq \frac{4}{3} \int_{1 / 4}^{3 / 4} \frac{u d u}{\operatorname{Length}\left(\beta_{u}\right)} \\
& \geq \frac{1}{12 \int_{1 / 4}^{3 / 4} \operatorname{Length}\left(\beta_{u}\right) u d u} \geq \frac{1}{12 \pi} .
\end{aligned}
$$

Putting everything together

$$
\frac{2 \pi}{\log 1 / r} \geq \frac{1}{12 \pi}
$$

So

$$
r \geq \exp \left\{-24 \pi^{2}\right\}
$$

This proves the theorem when $0<\zeta<1 / 4$.

So assume that $1>\zeta \geq 1 / 4$ and consider the circles $S_{u}$ centered at $\zeta$ of radius $u$. Note that

$$
M:=\max \left\{u: E \cap S_{u} \neq \emptyset\right\} \geq \zeta \geq m:=\min \left\{u: E \cap S_{u} \neq \emptyset\right\} .
$$

If $M-\zeta>1 / 8$, we consider circles centered at $\zeta$ with radius $\zeta<u<\zeta+1 / 8$. Then $E$ intersects every such circle, and we can repeat the same argument as above. The only difference arises for the estimate (2.1), because now only a portion of $\gamma_{u}$ lies schlichtly above $D(0,1)$. But the total length of $\gamma_{u}$ is comparable to the length of the part of $\gamma_{u}$ that lies above $D(0,1)$, i.e., there is a universal constant $C$, related to the angular measure of $S_{u} \cap D(0,1)$ about $\zeta$, such that

$$
\operatorname{Length}\left(\gamma_{u}\right) \leq C \text { Length }\left(\gamma_{u} \cap D(0,1)\right) .
$$

Likewise, if $\zeta-m>1 / 8$, then the same argument works.

So assume that $\zeta-1 / 8 \leq m<M \leq \zeta+1 / 8$. Then $E$ intersects $\partial \mathbb{D}$ in a point $a$ with $\operatorname{Re} a>47 / 128>1 / 8$. Therefore $E$ intersects the boundary $\partial R_{u}$ of each rectangle

$$
R_{u}=\{u<x<2 \zeta-u,-1-u<y<1+u\}, \quad u \in(0,1 / 8) .
$$


Now we can repeat the same argument as above, except that the function $g$ is replaced by $\log (\phi(f(z))-\zeta)$ where $\phi$ is a quasi-conformal map that fixes $\zeta$ and sends the rectangles $R_{u}$ to circles centered at $\zeta$ of radius $\zeta+u$. The conclusion follows because the distortion of $\phi$ is controlled by universal constants. In this case, one really needs to use Poletskii's inequality instead of Ohtsuka's result.

\section{An alternative approach using Beurling's CRIterion}

After this paper was written, we realized that one can do away with Poletskii's inequality altogether by using Beurling's criterion. For completeness, we decided to include both proofs.

First we recall Beurling's criterion for extremal metrics; see Ahl73. Theorem 4-4, page 61 .

Theorem 3.1 (Beurling's criterion). Let $\Gamma$ be a path family in $\mathbb{C}$. A Borel measurable function $\rho_{0}: \mathbb{C} \rightarrow[0,+\infty]$ is extremal (i.e., $\int_{\mathbb{C}} \rho_{0}^{2} d A \leq \int_{\mathbb{C}} \rho^{2} d A$ for every admissible $\rho$ ), if $\Gamma$ contains a subfamily $\Gamma_{0}$ with the following two properties:

(1) for every $\gamma \in \Gamma_{0}$ :

$$
\int_{\gamma} \rho_{0}|d z|=1
$$

(2) for every Borel measurable $h: \mathbb{C} \rightarrow[-\infty,+\infty]$ with the property that

$$
\int_{\gamma} h|d z| \geq 0
$$

for all $\gamma \in \Gamma_{0}$, we must have

$$
\int_{\mathbb{C}} h \rho_{0} d A \geq 0
$$

The proof of Beurling's theorem is a simple application of the Cauchy-Schwarz inequality.

An alternative proof of Theorem 1.1. We use the same notations as in Section 2. Again we assume first that $0<\zeta<1 / 4$ and define the curves $\alpha_{u}$ as before. We want a lower bound for the modulus of the path family $\Gamma$ of all such lifts $\alpha_{u}$. Recall also that, except for at most finitely many values of $u$ the paths $\alpha_{u}$ could be grouped so that $V_{n}=\bigcup_{u \in I_{n}} \alpha_{u}$ is an open simply connected set in $\mathbb{D}, n=1,2, \ldots, N$.

We claim that the following metric is extremal for $\Gamma$ :

$$
\rho_{0}(z)=\frac{\left|g^{\prime}(z)\right|}{\operatorname{Length}\left(\beta_{u}\right)} \quad \text { for } z \in V_{n} \cap \alpha_{u}
$$

and $\rho_{0}(z)=0$ otherwise. We let $\Gamma_{0}=\Gamma$ and check Beurling's criterion. Property (1) of Theorem 3.1 is clear. For property (2), assume that $h$ is a function as above, then, by the co-area formula,

$$
\int_{\mathbb{C}} h \rho_{0} d A=\int_{1 / 4}^{3 / 4} \frac{\int_{\alpha_{u}} h(z)|d z|}{u \operatorname{Length}\left(\beta_{u}\right)} d u \geq 0
$$


So, by co-area and (2.1)

$$
\begin{aligned}
\operatorname{Mod}(\Gamma) & =\int_{\mathbb{C}} \rho_{0}^{2} d A \\
& =\int_{1 / 4}^{3 / 4} \frac{\int_{\alpha_{u}}\left|g^{\prime}(z)\right||d z|}{u \operatorname{Length}\left(\beta_{u}\right)^{2}} d u \\
& =\int_{1 / 4}^{3 / 4} \frac{d u}{u \operatorname{Length}\left(\beta_{u}\right)} \\
& \geq \frac{1}{4} \frac{1}{\int_{1 / 4}^{3 / 4} \operatorname{Length}\left(\beta_{u}\right) u d u}>\frac{1}{4 \pi}
\end{aligned}
$$

This proves Theorem 1.1 when $0<\zeta<1 / 4$. The other cases are handled similarly. In the last case when $g$ is a quasi-regular map, the extremal metric must be defined with $\left|g^{\prime}(z)\right|$ replaced a.e. by

$$
\ell(z)=\inf _{|v|=1}|D g(z) v|
$$

where $D g$ is the differential matrix of $g$ and $v$ is a $2 \times 1$ vector in $\mathbb{R}^{2}$.

\section{ACKNOWLEDGMENTS}

We thank Don Marshall and David Drasin for helpful comments on a preliminary version of this paper.

\section{REFERENCES}

[Ahl73] Lars V. Ahlfors. Conformal invariants: topics in geometric function theory. McGrawHill Book Co., New York, 1973. McGraw-Hill Series in Higher Mathematics. MR0357743 $(50: 10211)$

[Mar89] Donald E. Marshall. A new proof of a sharp inequality concerning the Dirichlet integral. Ark. Mat., 27(1):131-137, 1989. MR1004727 (90h:30097)

[MSZ03] Jan Malý, David Swanson, and William P. Ziemer. The co-area formula for Sobolev mappings. Trans. Amer. Math. Soc., 355(2):477-492 (electronic), 2003. MR1932709 (2004a:46037)

[Oht70] Makoto Ohtsuka. Dirichlet problem, extremal length, and prime ends. Van Nostrand, 1970.

[Ric93] Seppo Rickman. Quasiregular mappings, volume 26 of Ergebnisse der Mathematik und ihrer Grenzgebiete (3) [Results in Mathematics and Related Areas (3)]. Springer-Verlag, Berlin, 1993. MR1238941 (95g:30026)

Department of Mathematics, Cardwell Hall, Kansas State University, Manhattan, KANSAS 66506

E-mail address: pietro@math.ksu.edu 